\title{
Search for Squarks and Gluinos in Single-Photon Events with Jets and Large Missing Transverse Energy in $p \bar{p}$ Collisions at $\sqrt{s}=1.8 \mathrm{TeV}$
}

B. Abbott, ${ }^{40}$ M. Abolins, ${ }^{37}$ V. Abramov, ${ }^{15}$ B. S. Acharya, ${ }^{8}$ I. Adam, ${ }^{39}$ D. L. Adams,${ }^{48}$ M. Adams, ${ }^{24}$ S. Ahn,${ }^{23}$ H. Aihara, ${ }^{17}$ G. A. Alves, ${ }^{2}$ N. Amos,${ }^{36}$ E. W. Anderson, ${ }^{30}$ R. Astur, ${ }^{42}$ M. M. Baarmand,${ }^{42}$ V. V. Babintsev, ${ }^{15}$ L. Babukhadia, ${ }^{16}$ A. Baden, ${ }^{33}$ V. Balamurali, ${ }^{28}$ B. Baldin, ${ }^{23}$ S. Banerjee,${ }^{8}$ J. Bantly, ${ }^{45}$ E. Barberis, ${ }^{17}$ P. Baringer ${ }^{31}$ J. F. Bartlett, ${ }^{23}$ A. Belyaev, ${ }^{14}$ S. B. Beri, ${ }^{6}$ I. Bertram, ${ }^{26}$ V. A. Bezzubov, ${ }^{15}$ P.C. Bhat,${ }^{23}$ V. Bhatnagar, ${ }^{6}$ M. Bhattacharjee, ${ }^{42}$ N. Biswas, ${ }^{28}$ G. Blazey, ${ }^{25}$ S. Blessing,${ }^{21}$ P. Bloom, ${ }^{18}$ A. Boehnlein, ${ }^{23}$ N. I. Bojko, ${ }^{15}$ F. Borcherding, ${ }^{23}$ C. Boswell, ${ }^{20}$ A. Brandt, ${ }^{23}$ R. Breedon, ${ }^{18}$ R. Brock, ${ }^{37}$ A. Bross, ${ }^{23}$ D. Buchholz,${ }^{26}$ V. S. Burtovoi, ${ }^{15}$ J. M. Butler, ${ }^{34}$ W. Carvalho, ${ }^{2}$ D. Casey,${ }^{37}$ Z. Casilum, ${ }^{42}$ H. Castilla-Valdez,${ }^{11}$ D. Chakraborty, ${ }^{42}$ S.-M. Chang,${ }^{35}$

S. V. Chekulaev, ${ }^{15}$ L.-P. Chen, ${ }^{17}$ W. Chen, ${ }^{42}$ S. Choi, ${ }^{10}$ S. Chopra ${ }^{36}$ B. C. Choudhary,${ }^{20}$ J. H. Christenson, ${ }^{23}$ M. Chung, ${ }^{24}$ D. Claes, ${ }^{38}$ A. R. Clark,${ }^{17}$ W. G. Cobau, ${ }^{33}$ J. Cochran, ${ }^{20}$ L. Coney, ${ }^{28}$ W. E. Cooper, ${ }^{23}$ C. Cretsinger ${ }^{41}$

D. Cullen-Vidal, ${ }^{45}$ M. A. C. Cummings,${ }^{25}$ D. Cutts,${ }^{45}$ O. I. Dahl,${ }^{17}$ K. Davis,${ }^{16}$ K. De,${ }^{46}$ K. Del Signore, ${ }^{36}$ M. Demarteau, ${ }^{23}$ D. Denisov, ${ }^{23}$ S. P. Denisov, ${ }^{15}$ H. T. Diehl,${ }^{23}$ M. Diesburg, ${ }^{23}$ G. Di Loreto, ${ }^{37}$ P. Draper,${ }^{46}$ Y. Ducros, ${ }^{5}$ L. V. Dudko, ${ }^{14}$ S. R. Dugad, ${ }^{8}$ A. Dyshkant,${ }^{15}$ D. Edmunds,,${ }^{37}$ J. Ellison, ${ }^{20}$ V. D. Elvira, ${ }^{42}$ R. Engelmann, ${ }^{42}$ S. Eno, ${ }^{33}$ G. Eppley, ${ }^{48}$ P. Ermolov, ${ }^{14}$ O. V. Eroshin, ${ }^{15}$ V. N. Evdokimov, ${ }^{15}$ T. Fahland,${ }^{19}$ M. K. Fatyga, ${ }^{41}$ S. Feher ${ }^{23}$ D. Fein, ${ }^{16}$ T. Ferbel, ${ }^{41}$ G. Finocchiaro, ${ }^{42}$ H. E. Fisk, ${ }^{23}$ Y. Fisyak, ${ }^{43}$ E. Flattum, ${ }^{23}$ G. E. Forden, ${ }^{16}$ M. Fortner, ${ }^{25}$ K. C. Frame, ${ }^{37}$ S. Fuess, ${ }^{23}$ E. Gallas, ${ }^{46}$ A. N. Galyaev, ${ }^{15}$ P. Gartung, ${ }^{20}$ V. Gavrilov, ${ }^{13}$ T. L. Geld, ${ }^{37}$ R. J. Genik II,${ }^{37}$ K. Genser,${ }^{23}$ C. E. Gerber, ${ }^{23}$ Y. Gershtein, ${ }^{13}$ B. Gibbard, ${ }^{43}$ B. Gobbi, ${ }^{26}$ B. Gómez, ${ }^{4}$ G. Gómez, ${ }^{33}$ P. I. Goncharov, ${ }^{15}$ J. L. González Solís, ${ }^{11}$ H. Gordon, ${ }^{43}$ L. T. Goss,${ }^{47}$ K. Gounder, ${ }^{20}$ A. Goussiou, ${ }^{42}$ N. Graf, ${ }^{43}$ P. D. Grannis, ${ }^{42}$ D. R. Green, ${ }^{23}$ H. Greenlee, ${ }^{23}$ S. Grinstein, ${ }^{1}$ P. Grudberg, ${ }^{17}$ S. Grünendahl,${ }^{23}$ G. Guglielmo, ${ }^{44}$ J. A. Guida, ${ }^{16}$ J. M. Guida, ${ }^{45}$ A. Gupta, ${ }^{8}$ S. N. Gurzhiev, ${ }^{15}$ G. Gutierrez, ${ }^{23}$ P. Gutierrez, ${ }^{44}$ N. J. Hadley, ${ }^{33}$ H. Haggerty, ${ }^{23}$ S. Hagopian, ${ }^{21}$ V. Hagopian, ${ }^{21}$ K. S. Hahn, ${ }^{41}$ R. E. Hall, ${ }^{19}$ P. Hanlet, ${ }^{35}$ S. Hansen, ${ }^{23}$ J. M. Hauptman,${ }^{30}$ D. Hedin, ${ }^{25}$ A. P. Heinson, ${ }^{20}$ U. Heintz, ${ }^{23}$ R. Hernández-Montoya, ${ }^{11}$ T. Heuring,${ }^{21}$ R. Hirosky, ${ }^{24}$ J. D. Hobbs, ${ }^{42}$ B. Hoeneisen,,${ }^{4} *$ J. S. Hoftun, ${ }^{45}$ F. Hsieh, ${ }^{36}$ Ting Hu, ${ }^{42}$ Tong Hu, ${ }^{27}$ T. Huehn, ${ }^{20}$ A. S. Ito ${ }^{23}$ E. James, ${ }^{16}$ J. Jaques,${ }^{28}$ S. A. Jerger ${ }^{37}$ R. Jesik, ${ }^{27}$ T. Joffe-Minor, ${ }^{26}$ K. Johns, ${ }^{16}$ M. Johnson, ${ }^{23}$ A. Jonckheere, ${ }^{23}$ M. Jones, ${ }^{22}$ H. Jöstlein, ${ }^{23}$ S. Y. Jun, ${ }^{26}$ C. K. Jung, ${ }^{42}$ S. Kahn, ${ }^{43}$ G. Kalbfleisch, ${ }^{44}$ D. Karmanov, ${ }^{14}$ D. Karmgard, ${ }^{21}$ R. Kehoe, ${ }^{28}$ M. L. Kelly, ${ }^{28}$ S. K. Kim, ${ }^{10}$ B. Klima,${ }^{23}$ C. Klopfenstein, ${ }^{18}$ W. Ko, ${ }^{18}$ J. M. Kohli, ${ }^{6}$ D. Koltick,${ }^{29}$ A. V. Kostritskiy, ${ }^{15}$ J. Kotcher, ${ }^{43}$ A. V. Kotwal, ${ }^{39}$ A. V. Kozelov, ${ }^{15}$ E. A. Kozlovsky, ${ }^{15}$ J. Krane, ${ }^{38}$ M. R. Krishnaswamy, ${ }^{8}$ S. Krzywdzinski, ${ }^{23}$ S. Kuleshov, ${ }^{13}$ S. Kunori, ${ }^{33}$

F. Landry, ${ }^{37}$ G. Landsberg, ${ }^{45}$ B. Lauer, ${ }^{30}$ A. Leflat, ${ }^{14}$ J. Li, ${ }^{46}$ Q. Z. Li-Demarteau, ${ }^{23}$ J. G. R. Lima, ${ }^{3}$ D. Lincoln, ${ }^{23}$ S. L. Linn, ${ }^{21}$ J. Linnemann, ${ }^{37}$ R. Lipton, ${ }^{23}$ F. Lobkowicz, ${ }^{41}$ S. C. Loken, ${ }^{17}$ A. Lucotte, ${ }^{42}$ L. Lueking, ${ }^{23}$ A. L. Lyon, ${ }^{33}$ A. K. A. Maciel, ${ }^{2}$ R. J. Madaras, ${ }^{17}$ R. Madden, ${ }^{21}$ L. Magaña-Mendoza, ${ }^{11}$ V. Manankov, ${ }^{14}$ S. Mani, ${ }^{18}$ H. S. Mao,${ }^{23, \dagger}$ R. Markeloff, ${ }^{25}$ T. Marshall, ${ }^{27}$ M. I. Martin, ${ }^{23}$ K. M. Mauritz,${ }^{30}$ B. May, ${ }^{26}$ A. A. Mayorov,${ }^{15}$ R. McCarthy, ${ }^{42}$ J. McDonald, ${ }^{21}$ T. McKibben, ${ }^{24}$ J. McKinley, ${ }^{37}$ T. McMahon, ${ }^{44}$ H. L. Melanson, ${ }^{23}$ M. Merkin, ${ }^{14}$ K. W. Merritt, ${ }^{23}$

C. Miao ${ }^{45}$ H. Miettinen, ${ }^{48}$ A. Mincer, ${ }^{40}$ C. S. Mishra, ${ }^{23}$ N. Mokhov, ${ }^{23}$ N. K. Mondal, ${ }^{8}$ H. E. Montgomery,${ }^{23}$ P. Mooney, ${ }^{4}$ M. Mostafa, ${ }^{1}$ H. da Motta, ${ }^{2}$ C. Murphy, ${ }^{24}$ F. Nang, ${ }^{16}$ M. Narain, ${ }^{23}$ V. S. Narasimham, ${ }^{8}$ A. Narayanan, ${ }^{16}$ H. A. Neal, ${ }^{36}$ J. P. Negret, ${ }^{4}$ P. Nemethy, ${ }^{40}$ D. Norman, ${ }^{47}$ L. Oesch, ${ }^{36}$ V. Oguri, ${ }^{3}$ E. Oliveira, ${ }^{2}$ E. Oltman, ${ }^{17}$ N. Oshima, ${ }^{23}$

D. Owen,${ }^{37}$ P. Padley, ${ }^{48}$ A. Para, ${ }^{23}$ Y. M. Park, ${ }^{9}$ R. Partridge, ${ }^{45}$ N. Parua, ${ }^{8}$ M. Paterno ${ }^{41}$ B. Pawlik, ${ }^{12}$ J. Perkins, ${ }^{46}$

M. Peters,${ }^{22}$ R. Piegaia, ${ }^{1}$ H. Piekarz,${ }^{21}$ Y. Pischalnikov, ${ }^{29}$ B. G. Pope,${ }^{37}$ H. B. Prosper ${ }^{21}$ S. Protopopescu, ${ }^{43}$ J. Qian, ${ }^{36}$ P.Z. Quintas, ${ }^{23}$ R. Raja ${ }^{23}$ S. Rajagopalan, ${ }^{43}$ O. Ramirez,${ }^{24}$ S. Reucroft, ${ }^{35}$ M. Rijssenbeek, ${ }^{42}$ T. Rockwell,${ }^{37}$ M. Roco,${ }^{23}$ P. Rubinov, ${ }^{26}$ R. Ruchti, ${ }^{28}$ J. Rutherfoord, ${ }^{16}$ A. Sánchez-Hernández, ${ }^{11}$ A. Santoro, ${ }^{2}$ L. Sawyer, ${ }^{32}$ R. D. Schamberger, ${ }^{42}$ H. Schellman, ${ }^{26}$ J. Sculli, ${ }^{40}$ E. Shabalina, ${ }^{14}$ C. Shaffer,${ }^{21}$ H. C. Shankar, ${ }^{8}$ R. K. Shivpuri, ${ }^{7}$ M. Shupe,${ }^{16}$ H. Singh, ${ }^{20}$ J. B. Singh, ${ }^{6}$ V. Sirotenko, ${ }^{25}$ E. Smith, ${ }^{44}$ R. P. Smith ${ }^{23}$ R. Snihur, ${ }^{26}$ G. R. Snow, ${ }^{38}$ J. Snow, ${ }^{44}$ S. Snyder, ${ }^{43}$ J. Solomon, ${ }^{24}$ M. Sosebee, ${ }^{46}$ N. Sotnikova, ${ }^{14}$ M. Souza, ${ }^{2}$ A. L. Spadafora, ${ }^{17}$ G. Steinbrück ${ }^{44}$ R. W. Stephens, ${ }^{46}$ M. L. Stevenson, ${ }^{17}$ D. Stewart, ${ }^{36}$ F. Stichelbaut,${ }^{42}$ D. Stoker,${ }^{19}$ V. Stolin, ${ }^{13}$ D. A. Stoyanova, ${ }^{15}$ M. Strauss,${ }^{44}$ K. Streets, ${ }^{40}$ M. Strovink, ${ }^{17}$ A. Sznajder, ${ }^{2}$ P. Tamburello, ${ }^{33}$ J. Tarazi, ${ }^{19}$ M. Tartaglia, ${ }^{23}$ T. L. T. Thomas,${ }^{26}$ J. Thompson, ${ }^{33}$ T. G. Trippe,${ }^{17}$ P. M. Tuts, ${ }^{39}$ V. Vaniev,${ }^{15}$ N. Varelas, ${ }^{24}$ E. W. Varnes, ${ }^{17}$ D. Vititoe, ${ }^{16}$ A. A. Volkov, ${ }^{15}$ A. P. Vorobiev, ${ }^{15}$ H. D. Wahl,${ }^{21}$ G. Wang, ${ }^{21}$ J. Warchol, ${ }^{28}$ G. Watts, ${ }^{45}$ M. Wayne, ${ }^{28}$ H. Weerts,${ }^{37}$ A. White, ${ }^{46}$ J. T. White,,${ }^{47}$ J. A. Wightman, ${ }^{30}$ S. Willis, ${ }^{25}$ S. J. Wimpenny, ${ }^{20}$ J. V. D. Wirjawan,${ }^{47}$ J. Womersley, ${ }^{23}$ E. Won,${ }^{41}$ D. R. Wood, ${ }^{35} \mathrm{Z}$. Wu $,{ }^{23,}{ }^{\dagger} \mathrm{H} . \mathrm{Xu},{ }^{45}$ R. Yamada, ${ }^{23}$ P. Yamin,,${ }^{43}$ T. Yasuda,${ }^{35}$ P. Yepes, ${ }^{48}$ K. Yip,${ }^{23}$ C. Yoshikawa, ${ }^{22}$ S. Youssef, ${ }^{21}$ J. Yu, ${ }^{23}$ Y. Yu, ${ }^{10}$ 
B. Zhang, ${ }^{23, \dagger}$ Y. Zhou, ${ }^{23, \dagger}$ Z. Zhou, ${ }^{30}$ Z. H. Zhu, ${ }^{41}$ M. Zielinski, ${ }^{41}$ D. Zieminska, ${ }^{27}$ A. Zieminski, ${ }^{27}$ E. G. Zverev, ${ }^{14}$ and A. Zylberstejn ${ }^{5}$

(D0 Collaboration)

\author{
${ }^{1}$ Universidad de Buenos Aires, Buenos Aires, Argentina \\ ${ }^{2}$ LAFEX, Centro Brasileiro de Pesquisas Físicas, Rio de Janeiro, Brazil \\ ${ }^{3}$ Universidade do Estado do Rio de Janeiro, Rio de Janeiro, Brazil \\ ${ }^{4}$ Universidad de los Andes, Bogotá, Colombia \\ ${ }^{5}$ DAPNIA/Service de Physique des Particules, CEA, Saclay, France \\ ${ }^{6}$ Panjab University, Chandigarh, India \\ ${ }^{7}$ Delhi University, Delhi, India \\ ${ }^{8}$ Tata Institute of Fundamental Research, Mumbai, India \\ ${ }^{9}$ Kyungsung University, Pusan, Korea \\ ${ }^{10}$ Seoul National University, Seoul, Korea \\ ${ }^{11}$ CINVESTAV, Mexico City, Mexico \\ ${ }^{12}$ Institute of Nuclear Physics, Kraków, Poland \\ ${ }^{13}$ Institute for Theoretical and Experimental Physics, Moscow, Russia \\ ${ }^{14}$ Moscow State University, Moscow, Russia \\ ${ }^{15}$ Institute for High Energy Physics, Protvino, Russia \\ ${ }^{16}$ University of Arizona, Tucson, Arizona 85721 \\ ${ }^{17}$ Lawrence Berkeley National Laboratory and University of California, Berkeley, California 94720 \\ ${ }^{18}$ University of California, Davis, California 95616 \\ ${ }^{19}$ University of California, Irvine, California 92697 \\ ${ }^{20}$ University of California, Riverside, California 92521 \\ ${ }^{21}$ Florida State University, Tallahassee, Florida 32306 \\ ${ }^{22}$ University of Hawaii, Honolulu, Hawaii 96822 \\ ${ }^{23}$ Fermi National Accelerator Laboratory, Batavia, Illinois 60510 \\ ${ }^{24}$ University of Illinois at Chicago, Chicago, Illinois 60607 \\ ${ }^{25}$ Northern Illinois University, DeKalb, Illinois 60115 \\ ${ }^{26}$ Northwestern University, Evanston, Illinois 60208 \\ ${ }^{27}$ Indiana University, Bloomington, Indiana 47405 \\ ${ }^{28}$ University of Notre Dame, Notre Dame, Indiana 46556 \\ ${ }^{29}$ Purdue University, West Lafayette, Indiana 47907 \\ ${ }^{30}$ Iowa State University, Ames, Iowa 50011 \\ ${ }^{31}$ University of Kansas, Lawrence, Kansas 66045 \\ ${ }^{32}$ Louisiana Tech University, Ruston, Louisiana 71272 \\ ${ }^{33}$ University of Maryland, College Park, Maryland 20742 \\ ${ }^{34}$ Boston University, Boston, Massachusetts 02215 \\ ${ }^{35}$ Northeastern University, Boston, Massachusetts 02115 \\ ${ }^{36}$ University of Michigan, Ann Arbor, Michigan 48109 \\ ${ }^{37}$ Michigan State University, East Lansing, Michigan 48824 \\ ${ }^{38}$ University of Nebraska, Lincoln, Nebraska 68588 \\ ${ }^{39}$ Columbia University, New York, New York 10027 \\ ${ }^{40}$ New York University, New York, New York 10003 \\ ${ }^{41}$ University of Rochester, Rochester, New York 14627 \\ ${ }^{42}$ State University of New York, Stony Brook, New York 11794 \\ ${ }^{43}$ Brookhaven National Laboratory, Upton, New York 11973 \\ ${ }^{44}$ University of Oklahoma, Norman, Oklahoma 73019 \\ ${ }^{45}$ Brown University, Providence, Rhode Island 02912 \\ ${ }^{46}$ University of Texas, Arlington, Texas 76019 \\ ${ }^{47}$ Texas A\&M University, College Station, Texas 77843 \\ ${ }^{48}$ Rice University, Houston, Texas 77005 \\ (Received 14 August 1998)
}

We search for new physics using events with one high transverse energy photon, two or more jets, and an apparent imbalance in transverse energy, in $p \bar{p}$ collisions at the Fermilab Tevatron at $\sqrt{s}=1.8 \mathrm{TeV}$. Such events are predicted for production of supersymmetric particles in some models. No excess is observed beyond expected background. For the parameter space of the minimal supersymmetric standard model with branching fraction $B\left(\tilde{\chi}_{2}^{0} \rightarrow \gamma \tilde{\chi}_{1}^{0}\right)=1$ and $m_{\tilde{\chi}_{2}^{0}}-m_{\tilde{\chi}_{1}^{0}}>20 \mathrm{GeV}$, we obtain a $95 \%$ confidence level lower mass limit of $310 \mathrm{GeV}$ for equal mass squarks and gluinos. The 
results are also interpreted in models with low energy gauge mediated supersymmetry breaking. [S0031-9007(98)08065-X]

PACS numbers: 14.80.Ly, 12.60.Jv, 13.85.Rm

We search for physics beyond the standard model (SM) using events with one high transverse energy $\left(E_{T}\right)$ photon, two or more jets, and large imbalance in transverse energy $\left(\mathscr{H}_{T}\right)$. We call these $\gamma \mathscr{H}_{T}+\geq 2$ jets events. This search is motivated by recent suggestions [1,2] that supersymmetry may result in signatures involving one or more photons together with multiple jets and large $\mathscr{E}_{T}$.

Supersymmetry introduces for every particle in the SM a supersymmetric partner differing in spin by one-half. $R$ parity, defined as +1 for SM particles and -1 for their superpartners, is assumed to be conserved in this analysis, such that supersymmetric particles are produced in pairs and the lightest supersymmetric particle (LSP) is stable. In the minimal supersymmetric standard model (MSSM), the gaugino-Higgsino sector (excluding gluinos) is described by four parameters: $M_{1}, M_{2}, \mu$, and $\tan \beta$, where $M_{1}$ and $M_{2}$ are the $\mathrm{U}(1)$ and $\mathrm{SU}(2)$ gaugino mass parameters, $\mu$ is the Higgsino mass parameter, and $\tan \beta$ is the ratio of the vacuum expectation values of the two Higgs doublets. Gaugino-Higgsino mixing gives four neutral mass eigenstates (neutralinos $\tilde{\chi}_{i}^{0}, i=1, \ldots, 4$ ) and two charged eigenstates (charginos $\tilde{\chi}_{i}^{ \pm}, i=1,2$ ). The radiative decay of $\tilde{\chi}_{2}^{0} \rightarrow \gamma \tilde{\chi}_{1}^{0}$ dominates in some regions (not excluded by the CERN $e^{+} e^{-}$collider LEP $Z$ data) of parameter space [3] assuming sfermions are heavy and has been proposed as an explanation [2] of a candidate event reported by the CDF Collaboration [4]. Assuming that $\tilde{\chi}_{1}^{0}$ is the LSP, then the production of $\tilde{\chi}_{2}^{0}$ will yield $\gamma \mathbb{E}_{T}+X$ events. The $\gamma \mathbb{E}_{T}+X$ events may also arise in low energy gauge mediated supersymmetry breaking (GMSB) models [1] in which the gravitino $(\tilde{G})$ is the LSP and the $\tilde{\chi}_{1}^{0}$ is the next-to-lightest supersymmetric particle (NLSP). In this case, $\tilde{\chi}_{1}^{0}$ is unstable and decays into a photon plus a gravitino $\left(\tilde{\chi}_{1}^{0} \rightarrow \gamma \tilde{G}\right)$.

In this Letter, we present the first search for physics beyond the $\mathrm{SM}$ in the channel $p \bar{p} \rightarrow \gamma \mathbb{E}_{T}+\geq 2$ jets. Because of large backgrounds from QCD processes, we do not consider events with less than two jets. The data used in this analysis were collected with the D0 detector [5] during the 1992-1996 Tevatron run at a center of mass energy of $\sqrt{s}=1.8 \mathrm{TeV}$ and represent an integrated luminosity of $99.4 \pm 5.4 \mathrm{pb}^{-1}$. The trigger requires one electromagnetic (EM) cluster with $E_{T}>15 \mathrm{GeV}$, one jet with $E_{T}>10 \mathrm{GeV}$, and $\mathscr{E}_{T}>14 \mathrm{GeV}\left(\mathbb{E}_{T}>10 \mathrm{GeV}\right.$ for about $10 \%$ of the data). Photons are identified via a two-step process: the selection of isolated EM energy clusters and the rejection of such clusters with any associated charged tracks. The EM clusters are selected from calorimeter energy clusters by requiring (i) at least 95\% of the energy to be deposited in the EM section of the calorimeter, (ii) the transverse and longitudinal shower profiles to be consistent with those expected for an EM shower, and (iii) the energy in an annular isolation cone with radius $\left[\mathcal{R} \equiv \sqrt{(\Delta \phi)^{2}+(\Delta \eta)^{2}}\right] 0.2$ to 0.4 around the cluster in $\eta-\phi$ space to be less than $10 \%$ of the EM energy in an $\mathcal{R}=0.2$ cone, where $\eta$ and $\phi$ are the pseudorapidity and azimuth, respectively. The EM clusters that have either a reconstructed track or a large number of hits in the tracking chamber along a road joining the cluster and the interaction vertex are vetoed. $\ddot{E}_{T}$ is determined from the energy deposition in the calorimeter within $|\eta|<4.5$.

To be selected as $\gamma \ddot{H}_{T}+\geq 2$ jets candidates, events are first required to have at least one identified photon with $E_{T}^{\gamma}>20 \mathrm{GeV}$ and pseudorapidity $\left|\eta^{\gamma}\right|<1.1$ or $1.5<$ $\left|\eta^{\gamma}\right|<2.0$ (the region with EM calorimeter coverage and good photon identification) and two or more jets reconstructed using the energy depositions in the calorimeter with cones of radius $\mathcal{R}=0.5$, having $E_{T}^{j}>20 \mathrm{GeV}$ and $\left|\eta^{j}\right|<2.0$. We refer to the events passing these requirements as the $\gamma+\geq 2$ jets sample. The $\mathbb{E}_{T}$ distribution of these events is shown in Fig. 1. We then require $\not_{T}>$ $25 \mathrm{GeV}$. A total of 318 events satisfies all requirements.

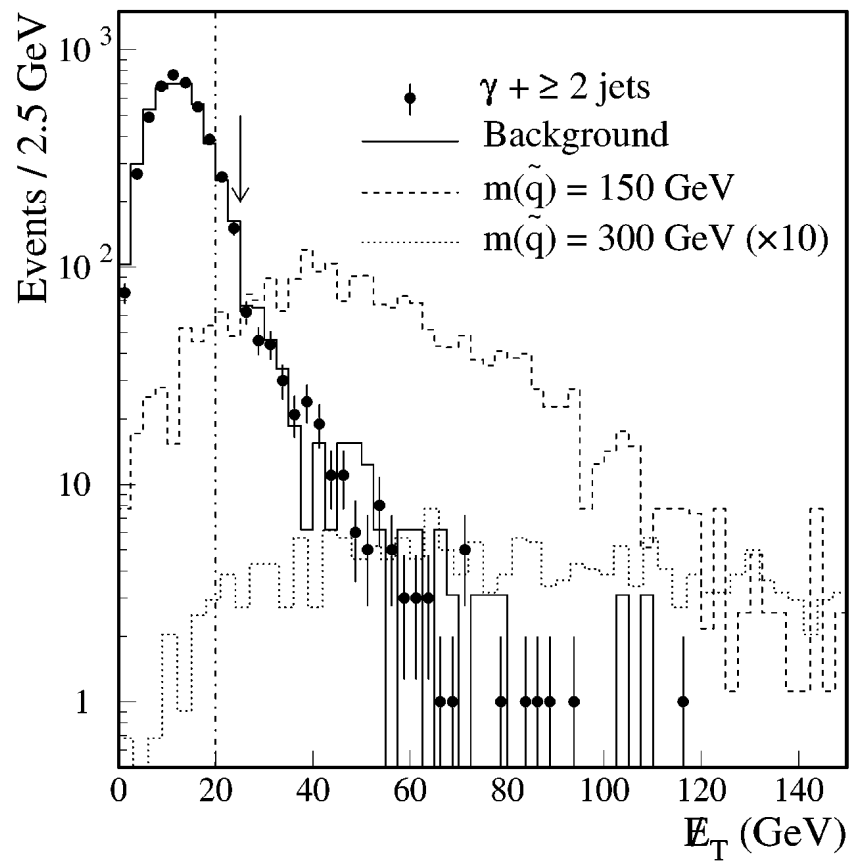

FIG. 1. The $\mathbb{E}_{T}$ distributions of the $\gamma+\geq 2$ jets and background events. The number of events in the background is normalized to the $\gamma+\geq 2$ jets sample for $\mathbb{E}_{T}<20 \mathrm{GeV}$, the region left of the dot-dashed line. The arrow indicates the $E_{T}>25 \mathrm{GeV}$ cut. Also shown are the distributions expected from supersymmetry for $m_{\tilde{q}}=m_{\tilde{g}}=150$ and $300 \mathrm{GeV}$. 
TABLE I. Number of observed $\gamma \mathscr{E}_{T}+n$ jets events $\left(N_{S}\right)$ together with the corresponding number of background events $\left(N_{B}\right)$ for $n \geq 2,3,4$, for three sets of cutoffs.

\begin{tabular}{|c|c|c|c|c|c|c|}
\hline \multirow{2}{*}{$\begin{array}{l}\text { No. } \\
\text { of jets }\end{array}$} & \multicolumn{2}{|c|}{$\begin{array}{c}\mathbb{E}_{T}>25 \mathrm{GeV} \\
\text { No } H_{T} \text { cut }\end{array}$} & \multicolumn{2}{|c|}{$\begin{array}{c}\mathscr{H}_{T}>25 \mathrm{GeV} \\
H_{T}>200 \mathrm{GeV}\end{array}$} & \multicolumn{2}{|c|}{$\begin{array}{c}\mathscr{E}_{T}>50 \mathrm{GeV} \\
\text { No } H_{T} \text { cut }\end{array}$} \\
\hline & $N_{S}$ & $N_{B}$ & $N_{S}$ & $N_{B}$ & $N_{S}$ & $N_{B}$ \\
\hline$n \geq 2$ & 318 & $320 \pm 30$ & 30 & $20 \pm 10$ & 43 & $65 \pm 15$ \\
\hline$n \geq 3$ & 70 & $70 \pm 15$ & 17 & $8 \pm 5$ & 11 & $10 \pm 5$ \\
\hline$n \geq 4$ & 8 & $10 \pm 5$ & 6 & $4 \pm 3$ & 1 & $3 \pm 3$ \\
\hline
\end{tabular}

The principal backgrounds are as follows: QCD direct photon and multijet events, where there is mismeasured $\mathbb{E}_{T}$ and a real or fake photon; $W(\rightarrow e \nu)+$ jets events, where the electron is misidentified as a photon and the neutrino gives rise to the $\mathbb{E}_{T}$; and $W(\rightarrow \ell \nu)+$ jets events (where $\ell=e, \mu, \tau$ ), in which one of the jets is misidentified as a photon. The background due to heavy quark production with a high $E_{T}$ photon is found to be negligible. The background from mismeasurement of $\not_{T}$ is estimated using events with one EM-like cluster that satisfies all photon criteria, except requirement (ii) on the shower profile. These events must also have two or more jets with $E_{T}^{j}>20 \mathrm{GeV}$ and $\left|\eta^{j}\right|<2.0$, making them similar to those of the $\gamma+\geq 2$ jets sample, and therefore of similar resolution in $\mathbb{E}_{T}$. The events in this background sample are normalized to the $\gamma+\geq 2$ jets sample for $\not_{T}<20 \mathrm{GeV}$, which provides an estimated background from $\mathbb{E}_{T}$ mismeasurement of $315 \pm 30$ events beyond $\mathbb{E}_{T}=25 \mathrm{GeV} . W+\geq 2$ jets events with $W \rightarrow e \nu$ can mimic $\gamma \mathscr{E}_{T}+\geq 2$ jets events if the electron is misidentified as a photon. This contribution is estimated using a sample of $e \mathbb{E}_{T}+\geq 2$ jets events that passes all our kinematic requirements, with the electron satisfying those defined for the photon. Electrons are selected from identified EM clusters that have matched tracks. Multiplying the probability $(0.0045 \pm 0.0008$, determined from $Z \rightarrow$ $e e$ data) that an electron is misidentified as a photon by the number of $e \not_{T}+\geq 2$ jets events yields a background of $4 \pm 1$ events. The $W(\rightarrow \ell \nu)+$ jets background is estimated using a data sample of $W(e \nu)+\geq 3$ jets events passing all kinematic requirements, with at least one of the jets satisfying those imposed on photons. Using the probability $(0.0007 \pm 0.0002$, determined using multijet data) that a jet is misidentified as a photon and the scale factor $N_{W(\rightarrow \ell \nu)+\geq 3 \text { jets }} / N_{W(\rightarrow e \nu)+\geq 3}$ jets (determined from Monte Carlo), we estimate a background of $1.0 \pm 0.3$ events. The background from $Z(\rightarrow \nu \nu)+\geq 3$ jets is negligible.

The number of observed events and the expected backgrounds are summarized in Table I, together with the breakdown by jet multiplicity. The $H_{T}$ distribution (defined as the scalar sum of the $E_{T}$ of all jets with $E_{T}^{j}>$ $20 \mathrm{GeV}$ and $\left|\eta^{j}\right|<2.0$ ) is shown in Fig. 2, for both $\gamma \mathbb{E}_{T}+\geq 2$ jets and background samples. Also given in Table I is the number of observed events and the expected background if the cutoff $H_{T}>200 \mathrm{GeV}$ is applied or if the $\mathbb{E}_{T}$ cutoff is raised to $50 \mathrm{GeV}$. In all three comparisons, the estimated number of background events agrees with the number of events observed in the data.

We interpret our results in terms of squark $(\tilde{q})$ and gluino $(\tilde{g})$ production in the context of supersymmetric models with a dominant $\tilde{\chi}_{2}^{0} \rightarrow \gamma \tilde{\chi}_{1}^{0}$ decay. We simulate $\tilde{q}$ and $\tilde{g}$ pair production and also production in association with $\tilde{\chi}_{i}^{0}$ and $\tilde{\chi}_{j}^{ \pm}$using the SPYTHIA program [6]. The MSSM parameters are set to $M_{1}=M_{2}=60 \mathrm{GeV}$, $\tan \beta=2$, and $\mu=-40 \mathrm{GeV}$. This set gives $m_{\tilde{\chi}_{1}^{0}}=$ $34 \mathrm{GeV}, m_{\tilde{\chi}_{2}^{0}}=60 \mathrm{GeV}$, and $B\left(\tilde{\chi}_{2}^{0} \rightarrow \gamma \tilde{\chi}_{1}^{0}\right)=1$. Slepton $(\tilde{\ell})$ and stop $\left(\tilde{t}_{1}\right)$ masses are set to $500 \mathrm{GeV}$ while sneutrino masses are fixed by the sum rule $m_{\tilde{\nu}}^{2}=m_{\tilde{\ell}}^{2}-$ $M_{W}^{2} \cos 2 \beta$. All other squarks are assumed to be degenerate in mass. Descriptions of superpartner decays can be found in Ref. [6]. However, we note that the mode $\tilde{q} \rightarrow q \tilde{\chi}_{2}^{0}$ dominates over the mode $\tilde{q} \rightarrow q \tilde{\chi}_{1}^{0}$ for the above values of supersymmetry parameters. Monte Carlo (MC) events are generated for three $\tilde{q}$ or $\tilde{g}$ mass possibilities: (i) equal mass $\tilde{q}$ and $\tilde{g}\left(m_{\tilde{q}}=m_{\tilde{g}}\right)$, (ii) heavy

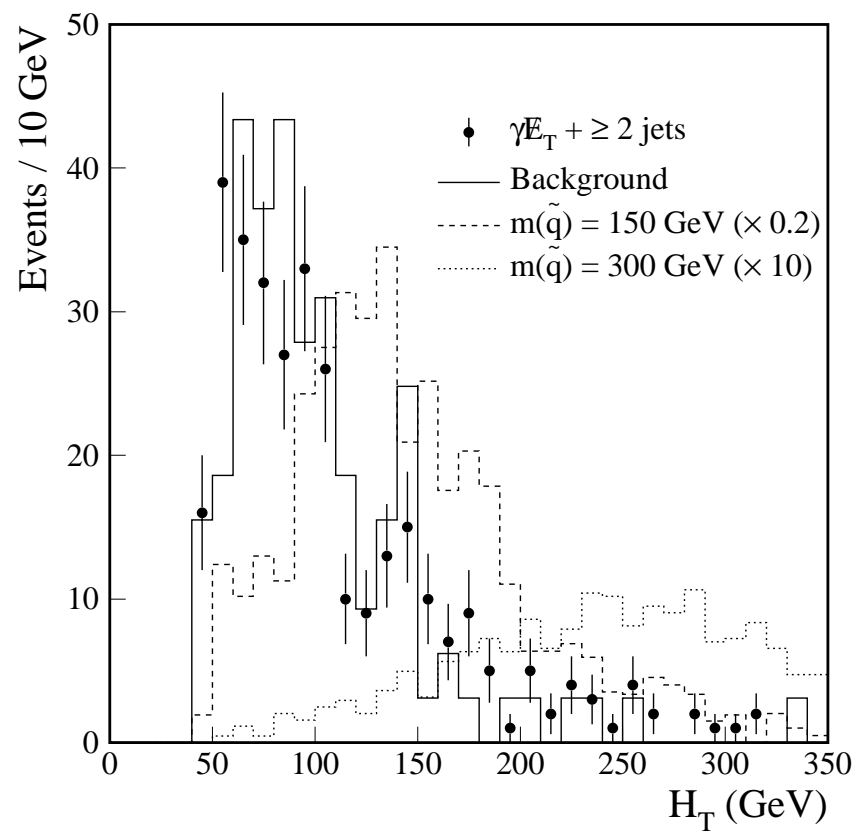

FIG. 2. The $H_{T}$ distributions of the $\gamma \mathscr{E}_{T}+\geq 2$ jets and background events. The expected distributions from supersymmetry are also shown for comparison. 
TABLE II. The percentages of events $\left(\epsilon_{0}\right)$ generated containing $\tilde{\chi}_{2}^{0}$ in the final state, and the efficiencies $\left(\epsilon_{S}\right)$ for their detection. The decrease in efficiency from $m_{\tilde{q} / \tilde{g}}=150$ to $200 \mathrm{GeV}$ is due to the change in the cuts as discussed in the text. The uncertainties are purely statistical.

\begin{tabular}{ccrcrrr}
\hline \hline$m_{\tilde{q} / \tilde{g}}$ & \multicolumn{2}{c}{$m_{\tilde{q}}\left(=m_{\tilde{g}}\right)$} & \multicolumn{2}{c}{$m_{\tilde{g}}\left(\ll m_{\tilde{q}}\right)$} & \multicolumn{2}{c}{$m_{\tilde{q}}\left(\ll m_{\tilde{g}}\right)$} \\
$(\mathrm{GeV})$ & $\epsilon_{0}(\%)$ & \multicolumn{1}{c}{$\epsilon_{S}(\%)$} & $\epsilon_{0}(\%)$ & \multicolumn{1}{c}{$\epsilon_{S}(\%)$} & \multicolumn{1}{c}{$\epsilon_{0}(\%)$} & \multicolumn{1}{c}{$\epsilon_{S}(\%)$} \\
\hline 150 & 66.2 & $15.1 \pm 0.8$ & 69.1 & $11.6 \pm 0.9$ & 60.0 & $16.8 \pm 1.1$ \\
200 & 62.3 & $7.9 \pm 0.6$ & 59.6 & $5.3 \pm 0.6$ & 53.8 & $9.5 \pm 0.9$ \\
250 & 59.6 & $14.8 \pm 0.8$ & 49.7 & $13.6 \pm 1.1$ & 55.4 & $14.8 \pm 1.1$ \\
300 & 56.1 & $21.5 \pm 1.0$ & 43.1 & $19.0 \pm 1.3$ & 55.4 & $22.1 \pm 1.2$ \\
350 & 51.8 & $22.8 \pm 1.1$ & 39.3 & $23.5 \pm 1.5$ & 52.7 & $26.6 \pm 1.4$ \\
400 & 46.7 & $23.5 \pm 1.1$ & 33.3 & $22.7 \pm 1.6$ & 54.3 & $25.8 \pm 1.3$ \\
\hline \hline
\end{tabular}

$\tilde{q}$ and light $\tilde{g}\left(m_{\tilde{q}} \gg m_{\tilde{q}}\right)$, and (iii) light $\tilde{q}$ and heavy $\tilde{q}\left(m_{\tilde{q}} \ll m_{\tilde{q}}\right)$. The $\mathbb{E}_{T}$ and $H_{T}$ distributions for $m_{\tilde{q}}=$ $m_{\tilde{g}}=150300 \mathrm{GeV}$ events are shown, respectively, in Figs. 1 and 2, where the MC distributions are scaled by the factors shown in parentheses. The distributions expected from supersymmetry differ considerably from those of the background. To increase the sensitivity to supersymmetry, we introduce an $H_{T}$ cutoff and maximize the $\epsilon_{S} / \delta N_{B}$ ratio by varying the $\mathbb{E}_{T}$ and $H_{T}$ cutoffs. Here $\epsilon_{S}$ is the efficiency for signal, and $\delta N_{B}$ is the uncertainty on the estimated number of background events. To ensure high efficiencies for both low and high $\tilde{q}$ and $\tilde{g}$ masses, the optimization is done for two MC points $m_{\tilde{q}}=m_{\tilde{g}}=150$ and $300 \mathrm{GeV}$. The optimum values are $\not_{T}>35 \mathrm{GeV}$ and $H_{T}>100 \mathrm{GeV}$ for $150 \mathrm{GeV}$, and $\mathbb{E}_{T}>45 \mathrm{GeV}$ and $H_{T}>220 \mathrm{GeV}$ for $300 \mathrm{GeV}$. The $\epsilon_{S} / \delta N_{B}$ results (a function of $\tilde{q} / \tilde{g}$ mass) for the two sets are equal near $200 \mathrm{GeV}$. We apply the cutoffs optimized for the $150 \mathrm{GeV}$ mass point to MC events with $\tilde{q}$ and $\tilde{g}$ masses below $200 \mathrm{GeV}$ and apply those optimized for the $300 \mathrm{GeV}$ mass point to masses of $200 \mathrm{GeV}$ or above. The number of events observed for these two sets of cutoffs are 60 and 5, with $75 \pm 17$ and $8 \pm 6$ events expected from background processes. We observe no excess beyond the standard model.

The $\epsilon_{S}$ for predictions from the supersymmetric models are given in Table II. MC studies show that the overall efficiency varies by $4 \%$ for different choices of $M_{1}, M_{2}$, $\tan \beta$, and $\mu$ that are consistent with $B\left(\tilde{\chi}_{2}^{0} \rightarrow \gamma \tilde{\chi}_{1}^{0}\right)=$
1 and $m_{\tilde{\chi}_{2}^{0}}-m_{\tilde{\chi}_{1}^{0}}>20 \mathrm{GeV}$ [2]. The total systematic error on the efficiency is $9 \%$, including uncertainties in photon identification efficiency $(7 \%)$, the choice of values of the supersymmetry parameters $(4 \%)$, and the jet energy scale $(3 \%)$. We set a $95 \%$ confidence level (C.L.) upper limit on $\sigma \times B \equiv \sigma\left(p \bar{p} \rightarrow \tilde{q} / \tilde{g} \rightarrow \tilde{\chi}_{2}^{0}+\right.$ $X) \times B\left(\tilde{\chi}_{2}^{0} \rightarrow \gamma \tilde{\chi}_{1}^{0}\right)$ using a Bayesian approach with a flat prior distribution for the signal cross section. The resulting upper limit is tabulated in Table III.

Figure 3 shows the limit for $m_{\tilde{q}}=m_{\tilde{g}}$, together with the theoretical cross section, calculated using SPYTHIA with the CTEQ3L parton distribution functions [7]. The renormalization scale is set to the average transverse energy of the outgoing partons in the calculation. The band represents the range of predictions obtained by varying the supersymmetry parameters with the constraints that $B\left(\tilde{\chi}_{2}^{0} \rightarrow \gamma \tilde{\chi}_{1}^{0}\right)=1$ and $m_{\tilde{\chi}_{2}^{0}}-m_{\tilde{\chi}_{1}^{0}}>20 \mathrm{GeV}$. The intersection of the limit with the lower edge of the band is at $\sigma \times B=0.38 \mathrm{pb}$, leading to a lower limit for equal mass $\tilde{q}$ and $\tilde{g}$ of $310 \mathrm{GeV}$ at the $95 \%$ C.L.

We vary the slepton mass from $500 \mathrm{GeV}$ to the lower experimental limit of about $80 \mathrm{GeV}$ [8] in the MC. For $m_{\tilde{q}}=$ $m_{\tilde{g}}=300 \mathrm{GeV} \mathrm{MC}$ events, the percentage $\epsilon_{0}$ increases by $25 \%$ which leads to an increase in the mass limit by $\sim 10 \mathrm{GeV}$. A light stop $\tilde{t}_{1}$ would modify $\tilde{q}$ and $\tilde{g}$ decays and would affect $\tilde{\chi}_{2}^{0}$ production. If $m_{\tilde{t}_{1}}$ is lowered from 500 to $80 \mathrm{GeV}$ (approximate lower experimental limit [9]), a $15 \%$ reduction in $\tilde{\chi}_{2}^{0}$ production is predicted which lowers the limit for equal mass $\tilde{q}$ and $\tilde{g}$ by about $6 \mathrm{GeV}$.

TABLE III. The theoretical cross sections $\sigma \times B$ and our measured 95\% C.L. upper limits on $\sigma \times B$. The predictions are calculated for $M_{1}=M_{2}=60 \mathrm{GeV}, \tan \beta=2$, and $\mu=-40 \mathrm{GeV}$.

\begin{tabular}{ccccccc}
\hline \hline & \multicolumn{5}{c}{$\begin{array}{c}\sigma \times B(p b) \\
m_{\tilde{q} / \tilde{g}}\end{array}$} & \multicolumn{2}{c}{$\begin{array}{c}m_{\tilde{q}}\left(=m_{\tilde{g}}\right) \\
\text { Theory }\end{array}$} & Limit & Theory & Limit & \multicolumn{2}{c}{$\begin{array}{c}m_{\tilde{q}}\left(\ll m_{\tilde{g}}\right) \\
\text { Theory }\end{array}$} & Limit \\
\hline 150 & 83.4 & 2.0 & 24.1 & 2.6 & 8.51 & 1.8 \\
200 & 12.1 & 1.1 & 3.48 & 1.6 & 1.59 & 0.9 \\
250 & 2.37 & 0.57 & 0.51 & 0.63 & 0.43 & 0.58 \\
300 & 0.53 & 0.39 & 0.12 & 0.44 & 0.12 & 0.38 \\
350 & 0.13 & 0.37 & 0.02 & 0.37 & 0.03 & 0.32 \\
400 & 0.04 & 0.36 & 0.01 & 0.37 & 0.01 & 0.32 \\
\hline \hline
\end{tabular}




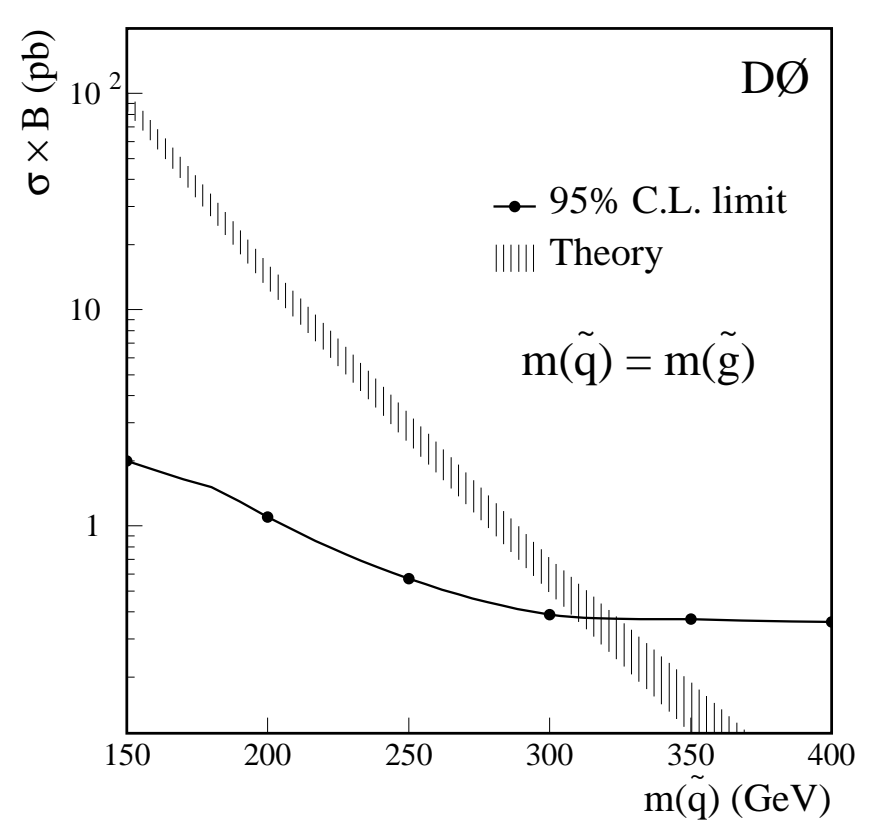

FIG. 3. The $95 \%$ C.L. upper limit on $\sigma \times B$ as a function of $m_{\tilde{q} / \tilde{g}}$, assuming equal $\tilde{q}$ and $\tilde{g}$ masses. The hatched band represents the range of expected cross sections for different sets of MSSM parameters; see text. The inflection below $200 \mathrm{GeV}$ in the limit curve is the intersection of the two curves using the two sets of optimized cutoffs discussed in the text.

Following the above procedure, we obtain a lower limit for $\tilde{g}(\tilde{q})$ mass of $240 \mathrm{GeV}$ when squarks (gluinos) are heavy. Again, these limits vary by approximately $10 \mathrm{GeV}$ if $\tilde{t}_{1}$ and/or sleptons are light.

The results of this analysis can also be interpreted in the GMSB models. In this case, every event from supersymmetry will have two photons in the final state for the prompt decay of the NLSP. This results in $\epsilon_{0}=$ $100 \%$ and leads to about a $50 \%$ increase in $\epsilon_{S}$. Using the above values of supersymmetry parameters, we set lower mass limits of $360,320,320 \mathrm{GeV}$ for the cases of $m_{\tilde{q}}=m_{\tilde{g}}, m_{\tilde{g}} \ll m_{\tilde{q}}$, and $m_{\tilde{q}} \ll m_{\tilde{g}}$, respectively. These results are insensitive to the choice of slepton and stop masses. Finally we note that the analysis is also sensitive to the delayed decay of the NLSP.

In summary, we have searched for an excess of $\gamma \mathbb{E}_{T}$ events with two or more jets in $p \bar{p}$ collisions at $\sqrt{s}=1.8 \mathrm{TeV}$. We find that the number of observed $\gamma \mathbb{E}_{T}+\geq 2$ jets events agrees well with that expected from background processes. Within the MSSM, with choices of parameters consistent with $B\left(\tilde{\chi}_{2}^{0} \rightarrow \gamma \tilde{\chi}_{1}^{0}\right)=1$ and $m_{\tilde{\chi}_{2}^{0}}-m_{\tilde{\chi}_{1}^{0}}>20 \mathrm{GeV}$, we obtain a $95 \%$ C.L. lower mass limit of $310 \mathrm{GeV}$ for equal mass squarks and gluinos and of $240 \mathrm{GeV}$ for squarks (gluinos) when gluinos (squarks) are heavy. These limits constrain the models discussed in Ref. [2] but do not exclude all of them.
These results are complementary to those [10] recently published by the LEP experiments.

We thank the staffs at Fermilab and collaborating institutions for their contributions to this work and acknowledge support from the Department of Energy and National Science Foundation (U.S.A.), Commissariat à L'Energie Atomique (France), Ministry for Science and Technology and Ministry for Atomic Energy (Russia), CAPES and CNPq (Brazil), Departments of Atomic Energy and Science and Education (India), Colciencias (Colombia), CONACyT (Mexico), Ministry of Education and KOSEF (Korea), and CONICET and UBACyT (Argentina).

*Visitor from Universidad San Francisco de Quito, Quito, Ecuador.

${ }^{\dagger}$ Visitor from IHEP, Beijing, China.

[1] S. Dimopoulos, S. Thomas, and J. D. Wells, Phys. Rev. D 54, 3283 (1996); S. Dimopoulos, M. Dine, S. Raby, and S. Thomas, Phys. Rev. Lett. 76, 3494 (1996); K. S. Babu, C. Kolda, and F. Wilczek, Phys. Rev. Lett. 77, 3070 (1996); J. L. Lopez, D. V. Nanopoulos, and A. Zichichi, Phys. Rev. Lett. 77, 5168 (1996); S. Ambrosanio et al., Phys. Rev. D 54, 5395 (1996); H. Baer, M. Brhlik, C.-H. Chen, and X. Tata, Phys. Rev. D 55, 4463 (1997).

[2] S. Ambrosanio et al., Phys. Rev. Lett. 76, 3498 (1996); Phys. Rev. D 55, 1372 (1997).

[3] H. Komatsu and J. Kubo, Phys. Lett. 157B, 90 (1985); H. E. Haber, G. L. Kane, and M. Quiros, Phys. Lett. 160B, 297 (1985); S. Ambrosanio and B. Mele, Phys. Rev. D 55, 1399 (1997); 56, 3157(E) (1997).

[4] CDF Collaboration, F. Abe et al., Phys. Rev. Lett. 81, 1791 (1998); Fermilab Report No. Fermilab-Pub-98/206E.

[5] D0 Collaboration, S. Abachi et al., Nucl. Instrum. Methods Phys. Res., Sect. A 338, 185 (1994).

[6] T. Sjöstrand, Comput. Phys. Commun. 82, 74 (1994); S. Mrenna, Comput. Phys. Commun. 101, 232 (1997). We used SPYTHIA version 5.7.

[7] H. L. Lai et al., Phys. Rev. D 51, 4763 (1995).

[8] ALEPH Collaboration, R. Barate et al., Phys. Lett. B 433, 176 (1998); DELPHI Collaboration, P. Abreu et al., CERN-EP/98-116; OPAL Collaboration, G. Abbiendi et al., CERN-EP/98-122; L3 Collaboration, M. Acciarri et al., CERN-PPE/97-130.

[9] D0 Collaboration, S. Abachi et al., Phys. Rev. Lett. 76, 2222 (1996); ALEPH Collaboration, R. Barate et al., Phys. Lett. B 434, 189 (1998); OPAL Collaboration, G. Abbiendi et al., CERN-EP/98-107; L3 Collaboration, M. Acciarri et al., CERN-PPE/97-135.

[10] ALEPH Collaboration, R. Barate et al., Phys. Lett. B 429, 201 (1998); OPAL Collaboration, G. Abbiendi et al., CERN-EP/98-143; DELPHI Collaboration, P. Abreu et al., CERN-EP/98-142; L3 Collaboration, M. Acciarri et al., CERN-EP/98-150. 